\title{
A Relative Research of the Software NHPP Reliability Based on Weibull Extension Distribution and Power Law Model
}

\author{
Tae-Hyun Yoo*1, Jung-Ki Lee' and Yun-JeongSeo² \\ 'Department of Science in Taxation, Namseoul University, South Korea; yth@nsu.ac.kr, leejk@nsu.ac.kr \\ 2School of Business Administration, Okayama Shoka University, Japan; yj-seo@po.osu.ac.jp
}

\begin{abstract}
Objectives: In this study, the comparative problem of reliability model by means of the Weibull extension distribution and power law pattern that completed out productivity of the software trustworthiness was proposed Methods/Statistical Analysis: In addition, the model choice founded on the mean rectangular error and measurement of fortitude for the proficient model were offered. Examination of the failure period influenced by the Weibull extension distribution and power law model was working for the recommending reliability model. The Laplace tendency test was offered for insurance about the failure period. Findings: In the deviation for the between of the predicted values with the actual observations, the shaping parameter from Weibull extension model regard as the best model and in terms of the prognostic power of the variance for the between the forecast values, the shaping parameter from Weibull extension model can be the efficacy model. From judgment of reliability, the situation of the reliability for the conditional work time shows that the case of the shaping parameter from Weibull extension model than shaping parameter from Weibull extension model, the shaping parameter from Weibull extension model, and power low model was revealed high reliability. Namely, the property of the reliability was reflected to subtle about the mission time. The resulting decisions were gained. In terms of deviation for between of the predicted values with the actual observations, the higher shaping model of Weibull extension model regard as the best model. The result of mean value functions has the tendency of non-decreasing form. The result of an intensity functions has the tendency non-increasing form. The case of the higher shaping model of Weibull extension model was judged more reliable model in reliability ground. Improvements/Applications: The software testing for the debugging to reduce cost in terms of the reliability from software is essential problem. From a research, the software developers must be considered for the growth model by the prior knowledge of the software to identify failure modes which can be able to help.
\end{abstract}

Keywords: Non-Homogeneous Poisson Process, Power Law Model, Weibull Extension Distribution

\section{Introduction}

Software failures for computer structures in our society can be influenced enormous damages. Therefore, software reliability from the software progress procedure can be the significant topic. The needs of the user necessities can be encountered the fee of testing. Thus, the reliability, fee and the release period are the significant item during the software progress procedure. Until present, numerous software reliability studies have been suggested. These studies for Non-Homogenous Poisson Process (NHPP) 1.2 from the fault detection procedure, if a fault happens, directly can be removed during the correcting procedure and has the hypothesis that new errors have not encountered.

In this study, the non-homogeneous Poisson process with an infinite number of faults from characteristics of the Weibull extension distribution and power law NHPP for the software reliability was presented.

*Author for correspondence 


\section{Basic Research}

\subsection{The Infinite NHPP Property using RVS}

The mean value feature and the intensity story $\frac{1,3}{3}$ through NHPP model were acknowledged next forms.

$$
m(t)=\int_{0}^{t} \lambda(s) d s, \frac{d h(t)}{d t}=\lambda(t)
$$

In the equation (1), $N(t)$ follows Poisson Probability Density Function (PDF) with the parameter $m(t)$. It can be embodied from following relationship ${ }^{4.5}$.

$$
p(N(t)=n)=\frac{[m(t)]^{n}}{n !} e^{-m(t)}, \boldsymbol{n}=\mathbf{O}, \mathbf{1}, \cdots, \infty .
$$

This period field model aimed at the NHPP can be well-defined for the likelihood of failure. The prototypical characteristic was contained the failure strength property $\lambda(t)$ and mean value property $m(t)$.

In infinite model, no new fault is supposed to happen at time of each repair. But, the real conditions at the point of repair new failure can behappened ${ }^{6,7}$.

Add to this condition, the Record Value Statistics (RVS) typical property can be specified from NHPP form. Therefore, property of mean value can be embodied from following relationship ${ }^{8-10}$.

$$
m(t)=-\ln (1-F(t))
$$

The equation (3) is mean value function about infinite failure NHPP typical from.

Therefore, the intensity function of infinite failure NHPP model may be embodied as the hazard property ( $h(t))$. Thus,

$$
\lambda(t)=m^{\prime}(t)=f(t) /(1-F(t))=h(t) .
$$

From pattern of the equation (4), $f(t)$ indicates PDF.

The expression $\left\{t_{n}, n=1,2, \ldots\right\}$ indicates the arrangement of the periods from between sequential software failures. Then $t_{n}$ denote the time of $\operatorname{among}(n-1)^{s t}$ and $n^{\text {th }}$ failure period. The expression $X_{n}$ denotes $n^{\text {th }}$ failure last period from the next condition.

$$
x_{n}=\sum_{k=1}^{n} t_{k} \quad\left(k=1,2, \cdots, n ; \quad 0 \leq x_{1} \leq x_{2} \leq \cdots \leq x_{n}\right)
$$

The likelihood pattern of $x_{1}, x_{2}, \ldots, x_{n}$ can be defined as follows ${ }^{1,10}$ :

$$
f_{X_{1}, X_{2}, \cdots, X_{n}}\left(x_{1}, x_{2}, \ldots, x_{n}\right)=L(\underline{x} \mid \Theta)=e^{-m\left(x_{n}\right)} \prod_{i=1}^{n} \lambda\left(x_{i}\right)
$$

Note that $\underline{x}=\left(x_{1}, x_{2}, x_{3}, \ldots, x_{n}\right)$ and $\Theta$ is the parameter space.

For the given arrangement of the software failure times $\left(x_{1}, x_{2}, \ldots, x_{n}\right)$, observations of the probability variable $\left(X_{1}, X_{2}, \ldots, X_{n}\right)$ can be assessed by means of the maximum likelihood method (MLE) ${ }^{1,9}$.

Consequently, reliability $\hat{R}\left(\tau \mid x_{n}\right)$ using the conditional work time was acknowledged as next form $\frac{910}{\text {. }}$

$\hat{R}\left(\tau \mid x_{n}\right)=e^{-\int_{x_{n}}^{x_{n}+\tau} \lambda(d) d t}=\exp \left[-\left\{m\left(\tau+x_{n}\right)-m\left(x_{n}\right)\right\}\right]$

Note that $\tau$ is a work time and $x_{n}$ is the preceding failure time.

\subsection{Weibull Extension Distribution}

$\mathrm{In}^{11}$ was proposed Weibull application model. The characteristic of this model has the bathtub-formed property for failure percentage function and asymptotically connected to the typical Weibull distribution. The new model also is the application of two parameter models was proposed by ${ }^{12}$ which can be used to model bathtub-formed property for failure percentage function. The relationships of PDF were specified as next form.

$$
\begin{gathered}
f(t)=\lambda \beta(\alpha t)^{\beta-1} \exp \left[(\alpha t)^{\beta}+\lambda \frac{1}{\alpha}\left(1-e^{(\alpha t)^{\beta}}\right)\right] \\
F(t)=1-\exp \left[-\lambda \frac{1}{\alpha}\left(e^{(\alpha t)^{\beta}}-1\right)\right]
\end{gathered}
$$

Note. $\alpha, \lambda, \beta>0, t \geq 0$.

The hazard function was acknowledged next form.

$$
h(t)=\frac{f(t)}{1-F(t)}=\lambda \beta(\alpha t)^{\beta-1} \exp \left[(\alpha t)^{\beta}\right]
$$

\subsection{Power Law NHPP Model}

The power law intensity function was known to next form $^{12,13}$.

$$
\lambda(t)=\alpha \beta t^{\beta-1}, \quad \alpha>0, \quad \beta>0, \quad t \in(0, \infty]
$$

The mean value function, using the intensity function from equation (11), is derived as follows:

$$
m(t \mid \Theta)=\int_{0}^{t} \lambda(s) d s=\alpha t^{\beta}
$$

Thus, the likeli hood patterncan bespecified as next property.

$$
L_{N H P P}(\Theta \mid \underline{x})=\left(\prod_{i=1}^{n} \alpha \beta x_{i}^{\beta-1}\right) \exp \left[-\alpha x_{n}{ }^{\beta}\right]
$$

Consequently, the log-likelihood assessment can be solved next form. 
$\ln L_{N H P P}(\Theta \mid \underline{x})=n \ln \alpha+n \ln \beta-(\beta-1) \sum_{i=1}^{n} \ln x_{i}-\alpha x_{n}{ }^{\beta}$

The estimation value $\hat{\alpha}_{M L E}$ and $\hat{\beta}_{M L E}$ should meet the next equations.

$$
\begin{gathered}
\frac{\partial \ln L_{N H P P}(\Theta \mid \underline{x})}{\partial \alpha}=\frac{n}{\alpha}-x_{n}{ }^{\beta}=0 \\
\frac{\partial \ln L_{N H P P}(\Theta \mid \underline{x})}{\partial \beta}=\frac{n}{\beta}-\sum_{i=1}^{n} \ln x_{i}-\alpha x_{n}{ }^{\beta} \ln x_{n}=0
\end{gathered}
$$

The reliability using the conditional work time the reliability is derived as follows:

$$
\hat{R}\left(\delta \mid x_{n}\right)=\exp \left[-\alpha\left(\delta+x_{n}\right)^{\beta}+\alpha x_{n}{ }^{\beta}\right]
$$

Note that $\delta$ is conditional work time.

\subsection{Comparison Criteria for the Efficient Model}

For investigating the usefulness of the planned model, the judgment principles was known to the next contain $\stackrel{9,10}{ }$.

The Mean Square Error (MSE) processes the deviation of between the forecast values with the actual values. It is well-defined as next form.

$$
M S E=\frac{\sum_{i=1}^{n}\left[m\left(x_{i}\right)-\hat{m}\left(x_{i}\right)\right]^{2}}{n-k}
$$

Note that $m_{i}$ is the whole cumulated quantity of the faults detected within period is $\left(0, t_{i}\right]$ and $\hat{m}_{i}$ growing amount of the failures at period $x_{i}$ can be gained from the correcting mean value is the projected, $n$ indicates the amount of realizing values and $k$ means the amount of parameters to can be assessed.

$R$ square $\left(R^{2}\right)$ can degree how effective the fit is in terms of the explaining from difference of the detected data. It is well-defined next property.

$$
R^{2}=1-\frac{\sum_{i=1}^{n}\left[m\left(x_{i}\right)-\hat{m}\left(x_{i}\right)\right]^{2}}{\sum_{i=1}^{n}\left(m\left(x_{i}\right)-\sum_{j=1}^{n} m\left(x_{j}\right) / n\right)^{2}}
$$

\section{Software Reliability Infinite NHPP Model Based on Weibull Extension Distribution}

The property of mean value characteristic $m(t)$ and intensity form $\lambda(t)$ of Weibull extension distribution software reliability model using the equation (9) and equation (10) are derived as follows:

$$
\begin{aligned}
& \lambda(t)=h(t)=\frac{f(t)}{1-F(t)}=\lambda \beta(\alpha t)^{\beta-1} \exp \left[(\alpha t)^{\beta}\right] \\
& m(t)=-\ln (1-F(t))=\frac{\lambda}{\alpha}\left(e^{(\alpha t)^{\beta}}-1\right)
\end{aligned}
$$

Thus, using the equation (6), the log likelihood function of Lomax model is derived as follows:

$$
\ln L_{N H P P}(\lambda, \alpha, \beta \mid \underline{x})=n \ln \lambda+n \ln \beta+(\beta-1) \sum_{i=1}^{n} \ln \left(\alpha x_{i}\right)+\sum_{i=1}^{n}\left(\alpha x_{i}\right)^{\beta}-\frac{\lambda}{\alpha}\left(e^{\left(\alpha x_{n}\right)^{\beta}}-1\right)
$$

Maximizing the equation (22) for the fixed value of $\beta(0.5,1.0,1.5)$ with respect to parameters $(\lambda, \alpha)$, the next conditions must be satisfied.

$$
\begin{gathered}
\frac{\partial \ln L_{N H P P}(\lambda, \alpha \mid \underline{x})}{\partial \lambda}=\frac{n}{\lambda}-\frac{1}{\alpha}\left(e^{\left(\alpha x_{n}\right)^{\beta}}-1\right)=0 \\
\frac{\partial \ln L_{N H P P}(\lambda, \alpha \mid \underline{x})}{\partial \alpha}=(\beta-1)\left(\frac{n}{\alpha}\right)+\beta \alpha^{\beta} \sum_{i=1}^{n} x_{i}^{\beta}+\frac{\lambda}{\alpha^{2}}\left(e^{\alpha x_{n}}-1-\alpha^{\beta} \beta x_{n}^{\beta} e^{\left(\alpha x_{n}\right)^{\beta}}\right)=0
\end{gathered}
$$

The reliability using the conditional work time can be derived as next property.

$$
\hat{R}\left(\delta \mid x_{n}\right)=\exp \left[-\left\{m\left(\delta+x_{n}\right)-m\left(x_{n}\right)\right\}\right]
$$

Using conditional work time $(\delta)$, reliability can be embodied from following relationship.

\section{Data Analysis for Software Failure Time}

In this chapter, the characteristics of Weibull extension distribution were analyzed by means of software failures time information ${ }^{14}$ using data given in Table 1 . The trend test $\frac{13,15}{}$ must is offered about the data for the trust models. Thus, the data analysis using Laplace trend test is recycled in this paper. The Laplace factor is indicated in between 2.0 and -2.0 in Figure 1, the reliability growth shows the properties. Thus, it is allowed to evaluate the reliability ${ }^{12}$ using proposed data.

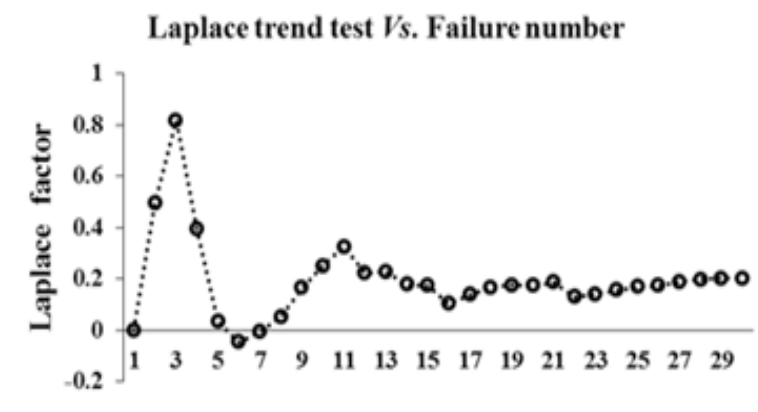

Failure number

Figure 1. Trend test using Laplace factor. 
Table 1. Software failure time information for proposed model.

\begin{tabular}{|l|l|l|l|}
\hline $\begin{array}{l}\text { Failure } \\
\text { number }\end{array}$ & $\begin{array}{l}\text { Failure time } \\
\text { (hours) }\end{array}$ & $\begin{array}{l}\text { Failure } \\
\text { Number }\end{array}$ & $\begin{array}{l}\text { Failure time } \\
\text { (hours) }\end{array}$ \\
\hline 1 & 0.479 & 16 & 10.771 \\
\hline 2 & 0.745 & 17 & 10.906 \\
\hline 3 & 1.022 & 18 & 11.183 \\
\hline 4 & 1.576 & 19 & 11.779 \\
\hline 5 & 2.61 & 20 & 12.536 \\
\hline 6 & 3.559 & 21 & 12.973 \\
\hline 7 & 4.252 & 22 & 15.203 \\
\hline 8 & 4.849 & 23 & 15.64 \\
\hline 9 & 4.966 & 24 & 15.98 \\
\hline 10 & 5.136 & 25 & 16.385 \\
\hline 11 & 5.253 & 26 & 16.96 \\
\hline 13 & 6.996 & 28 & 17.60 \\
\hline 14 & 8.170 & 29 & 18.122 \\
\hline 15 & 8.863 & 30 & 18.735 \\
\hline
\end{tabular}

The estimation value of the parameters for the proposed model was used the maximum likelihood technique. In this study, the mathematical alteration documents (Failure time (hours) $\times 0.1$ ) for simplify the parameter approximation was used. An outcome of the parameter approximation was obtained from the Table 2. These designs, resolving mathematically, a first values is given from 0.001 to 20.0 and permitted values for the measurement about an intermission was given to $10^{-5}$ by means of inspection for acceptable convergent using $C$-language, was achieved repetition of 100 times.
An outcomes for the parameter estimation using the MSE and coefficient of determination $\left(R^{2}\right)$ were exhibited in Table $2^{12}$.

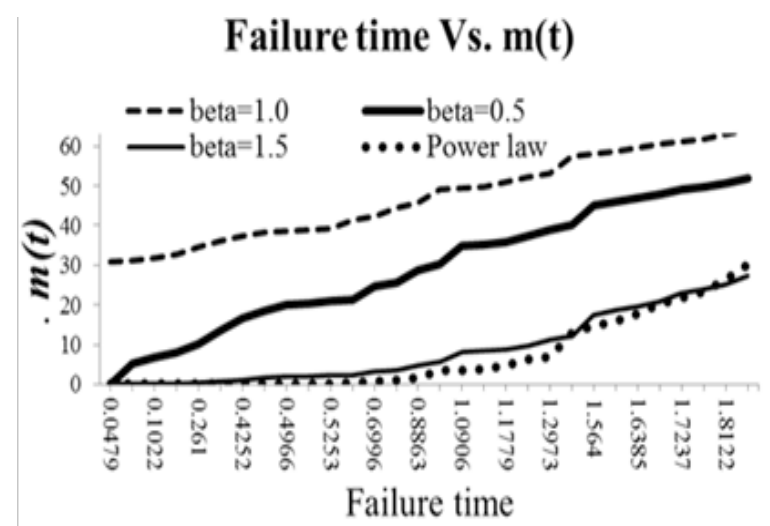

Note. In the figure 2, beta is $\beta$.

Figure 2. Property of mean value function about proposed model

In the software model comparison from Table 2, MSE shows that the case of shaping parameter $\beta=1.5$ (from Weibull extension model) than power low model and $\beta=0.5$ or $\beta=1.0$ (from Weibull extension model) has a small value. Therefore, the case of a shaping parameter $\beta=1.5$ (from Weibull extension model) is appreciably better than other considering the shaping parameters (from Weibull extension model) and power low model. But, $\mathrm{R}^{2}$ shows that the case of a shaping parameter $\beta=1.0$ (from Weibull extension model) than other the cases has high value. Thus, the case of a shaping parameter $\beta=1.0$

Table 2. Estimated value of $M L E, M S E, R^{2}$ about power law and Weibull extension distribution model.

\begin{tabular}{|c|c|c|c|c|c|}
\hline \multirow{3}{*}{$\begin{array}{l}\text { Power } \\
\text { low } \\
\text { model }\end{array}$} & \multirow{2}{*}{\multicolumn{3}{|c|}{$\begin{array}{l}\text { Maximum likelihood estimation } \\
\text { MSE } \\
R^{2}\end{array}$}} & \multicolumn{2}{|c|}{ Model comparison } \\
\hline & & & & & \\
\hline & \multicolumn{2}{|c|}{$\hat{\alpha}_{M L E}=2.5250$} & $\hat{\beta}_{M L E}=3.9422$ & 92.945 & 0.7845 \\
\hline \multirow{3}{*}{$\begin{array}{l}\text { Weibull } \\
\text { extension } \\
\text { model }\end{array}$} & \multirow{3}{*}{$\begin{array}{l}\text { Shape } \\
\text { parameter } \\
\text { (fixed) }\end{array}$} & $\beta=0.5$ & $\begin{array}{l}\hat{\alpha}_{M L E}=0.5992 \\
\hat{\lambda}_{M L E}=16.9349\end{array}$ & 253.812 & 0.9887 \\
\hline & & $\beta=1.0$ & $\begin{array}{l}\hat{\alpha}_{M L E}=0.0683 \\
\hat{\lambda}_{M L E}=15.0109\end{array}$ & 1073.913 & 0.9898 \\
\hline & & $\beta=1.5$ & $\begin{array}{l}\hat{\alpha}_{M L E}=0.6775 \\
\hat{\lambda}_{M L E}=6.3791\end{array}$ & 57.231 & 0.8922 \\
\hline
\end{tabular}

Note: $M L E$ : Maximum likelihood estimation.

$M S E$ : Mean square error.

$R^{2}$ : Coefficient of determination. 
(from Weibull extension model) than other the shaping parameters can bethe efficacy model. Ultimately, from the deviation for the between of the predicted values with the actual observations, the shaping parameter $\beta=1.5$ (from Weibull extension model) regard as the best model and in terms of the prognostic power of the variance for the between the forecast values, the shaping parameter $\beta=1.0$ (from Weibull extension model) model can be the efficacy model.

The result of mean value functions was listed in Figure 2. From this figure, the propertys of mean value characteristic have the style of the non-declining form. But, the result of intensitycharacteristicislisted in Figure 3. From this figure, a case of $\beta=0.5$ (from Weibull extension model), apattern of the intensity functions has the tendencynon-increasing form, the case of $\beta=1.0$ (from Weibull extension model)for a pattern of the intensity functions has nearlyconstant form, the case of $\beta=1.5$ (from Weibull extension model) and power lowmodel have the trend of the growing form.

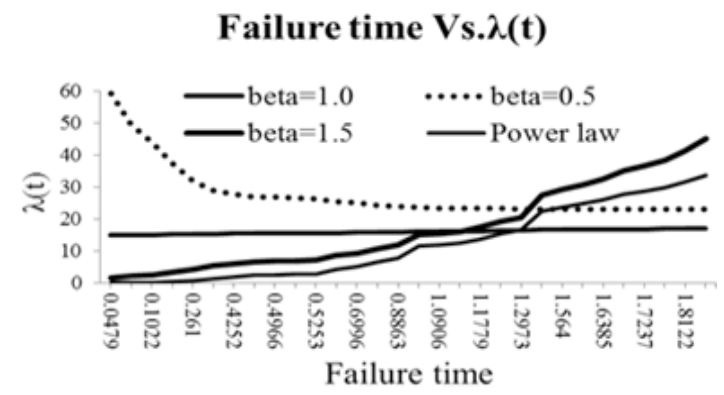

Figure 3. Property of intensity function about proposed model

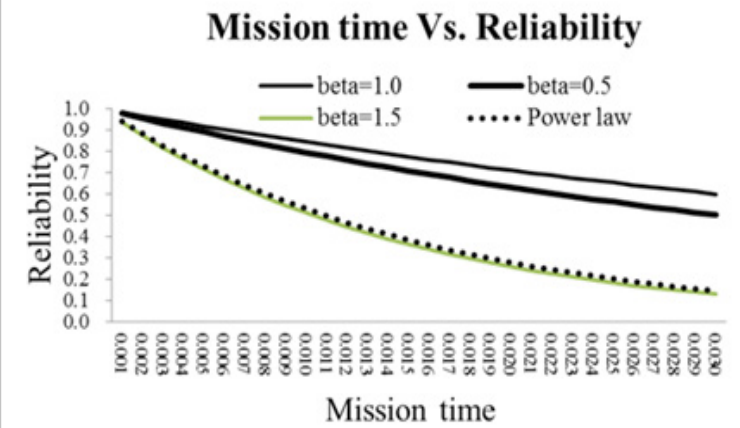

Figure 4. Comparison reliability for each model

In Figure 4, from judgment of reliability, the situation of a reliability for the conditional work time (mission time), shows that the case of the shaping parameter $\beta=1.0$ (from Weibull extension model) than shaping parameter $\beta=0.5$ (from Weibull extension model), the shaping parameter $\beta=0.5$ (from Weibull extension model), and power low model was revealed high reliability. Namely, the property of the reliability was reflected to subtle circumstance about the mission time.

Consequently, acase aboutthesmallershaping parameter (from Weibull extension model) was judged more reliable model in this field using Weibull extension distribution.

\section{Conclusion}

In this study, the comparative problem for the reliability model with the Weibull extension distribution and power law which made out efficiency for the software reliability was proposed.

In this study, the resulting decisions were gained.

In terms of deviation for between of the predicted values with the actual observations, the higher shaping model of Weibull extension model regard as the best model. The result of a mean value functions has the tendency of non-decreasing form. The result of an intensity functions has the tendency non-increasing form.

The case of the higher shaping model of Weibull extension model was judged more reliable model in reliability ground. The substitute homework for this zone will be appreciated study.

\section{Acknowledgment}

Funding for this paper was provided by Namseoul University

\section{References}

1. Gokhale SS, Trivedi KS. A Time/Structure Based Software Reliability Model, Annals of Software Engineering. 1998; 8(1):85-121.

2. Goel AL, Okumoto K. Time Dependent Error - Detection Rate Model for Software Reliability and Other Performance Measure, IEEE Transaction on Reliability. 1979; R-28(3):206-11.

3. Yamada S, Ohba H. S-Shaped Software Reliability Modeling for Software Error Detection, IEEE Transaction on Reliability. 1983; R-32(5):475-84.

4. Zhao M. Change-Point Problems in Software and Hardware Reliability, Communication Statistics Theory and Methods. 1993; 22(3):757-68. 
5. Shyur H-J. A Stochastic Software Reliability Model with Imperfectde Bugging and Change-Point, Journal of System Software. 2003; 66(2):135-41.

6. Pham H, Zhang X. NHPP Software Reliability and Cost Modelswith Testing Coverage, European Journal of Operational Research. 2003; 145(2):445-54.

7. Huang CY. Performance Analysis of Software Reliability Growth Models with Testing-Effort and Change-Point, Journal of System Software. 2005; 76(2):181-94.

8. Kuei-Chen C, Yeu-Shiang H, Tzai-Zang L. A Study of Software Reliability Growth from the Perspective of Learning Effects, Reliability Engineering and System Safety. 2008; 93(10):1410-21.

9. You T-H.The Infinite NHPP Software Reliability Model Based on Monotonic Intensity Function, Indian Journal of Science and Technology. 2015 Jul; 8(14):1-7.

10. Kuo L, Yang TY. Bayesian Computation of Software Reliability, Journal of the American Statistical Association. 1996; 91(434):763-73.
11. Fotouh SAEl, Nassar MMA. Estimation for the Parameters of the Weibull Extension Model Based on Generalized Order Statistics, International Journal of Contemporary Mathematics, Sciences. 2011; 6(36):1749 -17.

12. Xie M, Tang Y, Goh TN. A Modified Weibull Extension with Bathtub-Shaped Failure Rate Function, Reliability Engineering and System Safety. 2011; 76(3):279-85.

13. Kim H-C. The Property of Learning Effect Based on Delayed Software S-Shaped Reliability Model using Finite NHPP Software Cost Model, Indian Journal of Science and Technology. 2015 Dec; 8(34):1- 7.

14. Hayakawa Y, Telfar G. Mixed Poisson-Type Processes with Application in Software Reliability, Mathematical and Computer Modelling. 2000; 31(10-12):151-6.

15. Kim H-C. A Performance Analysis of Software Reliability Modelusing Lomax and Gompertz Distribution Property, Indian Journal of Science and Technology. 2016 May; $9(20): 1-6$. 